The Effect of COVID-19 Anxiety on General Health: The Role of COVID-19 Coping

\author{
*Murat Y1ldırımª, b; Ömer Akgülc; Ekmel Geçer ${ }^{\mathrm{c}}$

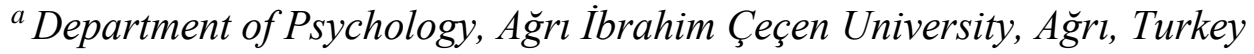 \\ ${ }^{b}$ Department of Neuroscience, Psychology and Behaviour, University of Leicester, \\ Leicester, $U K$ \\ ${ }^{c}$ Department of Psychology, University of Health Sciences, Istanbul, Turkey
}

Cite as: Yıldırım, M., Akgül, Ö., \& Geçer, E. (2020). The Effect of COVID-19 Anxiety on General Health: The Role of COVID-19 Coping. PsyArXiv, 1-24. Doi:

10.31234/osf.io/h8w9e

Authors note

*Correspondence: Dr Murat Yıldırım, Ağrı İbrahim Çeçen University, Department of Psychology, Faculty of Science and Letters, Erzurum Yolu 4 Km 04100, Merkez, Ağr1, Turkey; tel: +904722159863, email: muratyildirim@agri.edu.tr 


\begin{abstract}
Background and Objectives: The Coronavirus Disease 2019 (COVID-19) epidemic has unprecedently affected many aspects of people's lives including their health. This study examined the mediating effect of COVID-19 coping strategies on the relationship between COVID-19 anxiety and general health.
\end{abstract}

Design: Cross-sectional, correlational study.

Methods: Participants (70\% females) included 4,624 adults $\left(M_{\mathrm{age}}=30.29 \pm 10.97\right.$ years $)$ collected through online survey.

Results: Exploratory factor analysis yielded a one-factor structure for the COVID-19 Anxiety Scale, COVID-19 Coping Scale, and General Health Scale which exhibited good internal consistency reliability. Findings showed that COVID-19 anxiety was negatively related with COVID-19 coping and general health. COVID-19 coping had a positive relationship with general health. Hierarchical multiple regression analyses revealed that COVID-19 coping partially mediated the relationship between COVID-19 anxiety and general health.

Conclusions: These findings shed more light on the mechanism underlying between COVID19 anxiety and health. The findings are important for tailoring and implementing copingbased intervention strategies to reduce the impact of COVID-19 anxiety on general health. Keywords. COVID-19 anxiety, COVID-19 coping, General health, Coronavirus, Turkey 


\section{The Effect of COVID-19 Anxiety on General Health: The Role of COVID-19 Coping Strategies}

COVID-19 pandemic has spread around the world exponentially since it first appeared in China on December 2019. The virus has become a major health concern affecting almost all aspects of individuals lives. By 6 June 2020, statistics show that there were more than 6,850,000 confirmed COVID-19 cases worldwide, including 169,218 in Turkey, and more than 398,000 deaths (Center for Systems Science and Engineering, 2020). COVID-19 pandemic undoubtedly creates a general atmosphere of anxiety, fear, uncertainty, and insecurity (Ahorsu et al., 2020) that need to be psychologically investigated via well-designed studies in order to better comprehend its negative impacts on individuals' health. People adopt various personal strategies to cope with such challenging situations to protect their health. As such, this study examined the mediating role of COVID-19 coping strategies on the relationship between COVID-19 anxiety and general health.

By definition, anxiety is the natural waring system of the body representing the likelihood of a potential danger and action used to address it (Hooley, Butcher, Matthew, \& Mineka, 2016). Anxiety frequently occurs in the face of a new and unknow situation. Current COVID-19 pandemic and earlier outbreaks like SARS in 2003 and MERS-CoV in 2014 resulted in a great amount of psychological distress among people in infected areas. Studies demonstrated that negative effects of psychological responses (i.e., anxiety, depression, stress, fear) affect health and well-being of people in times of health crisis (Pappas, Kiriaze, Giannakis, \& Falagas, 2009; Wu, Chan, \& Ma, 2005).

Due to COVID-19, mental health illnesses including anxiety, depression, stress, and sleep disorder have become prevalent problems across the world. People who are under the 
high risk of infection, stress, and burnout can develop common mental health problems such as anxiety, depression, and fear (Bao, Sun, Meng, Shi, \& Lu, 2020). There have been various studies that have investigated the prevalence of psychological distress among different populations during pandemic. For instance, in Italy the prevalence rates of mental health problems have been reported as $49.38 \%$ for post-traumatic stress disorders, $24.73 \%$ for depression, $21.90 \%$ for anxiety, and $19.80 \%$ for perceived stress among healthcare workers (Rossi et al., 2020). Among general population in China, the prevalence of depressive symptoms, anxiety, and stress ranging from moderate to severe were respectively $16.5 \%$, $28.8 \%$, and $8.1 \%$ (Wang et al., 2020). High rates of anxiety, depression, stress, and other negative emotions have also been reported in the UK (The Academy of Medical Sciences, 2020).

Recent research has documented various contributing factors to psychosocial and physical health such as demographic, psychological, and behavioral factors during outbreaks. These factors include optimism-pessimism, psychological flexibility, hope, resilience, fear, vulnerability, perceived risk, and preventive behaviors (Arslan, Yıldırım, Tanhan, Buluş, \& Allen, 2020; Yıldırım, \& Arslan, 2020; Yıldırım, Geçer, \& Akgül, 2020). Stress management strategies have found to reduce maladaptive coping strategies including avoidance, antagonistic confrontation and self-blame which have been linked with worse mental health outcomes (Maunder et al., 2006; Sim et al., 2010). Despite these studies on various indices of psychological health, there is a knowledge gap linking to mechanism underlying the relationship between anxiety and psychological health in general population during COVID19 pandemic.

\section{Mediating effect of coping}


Psychological research has largely focused on negative states, their predictive factors, correlates, and consequences of COVID-19 on human health and life. Psychological strengths like coping strategies have been relatively neglected in addressing psychological distress during outbreaks. According to the transactional model, stress occurs when environmental or internal demands are appraised by a person as exceeding or taxing their ability to cope (Folkman, 2008; Lazarus \& Folkman, 1984). Theoretical approaches of coping emphasis strategies used to reduce distress. Coping has been researched in numerous environments and contexts (Carver, Scheier, \& Weintraub, 1989; Y1ldırım, 2019).

During current and past infectious disease outbreak, people have used various coping strategies to deal with challenges steamed from the diseases. While those who adopt adaptive coping styles can effectively overcome challenges and obstacles during outbreaks, others may suffer from psychopathological problems. A small number of studies have investigated the role of coping construct in the context of COVID-19. In a systematic review and narrative synthesis of the available evidence on contagious diseases, Chew et al. (2020) reported that people use a wide range of coping strategies (i.e., problem focused-coping, avoidance, seeking social support, and positive appraisal of the situation) to deal with negative psychological outcomes resulted from outbreaks such as fear, anxiety, depression, posttraumatic stress disorders, anger, grief and loss, guilt, and stigmatization. Studies on Philippine teachers' and students' practices to cope with anxiety during COVID-19 outbreak found that information seeking, preventive behaviors, spending time with family, seeking spiritual guidance, talking with friends online, using social media, playing online games, avoiding media news about COVID-19 fatalities, and other coping mechanism were among the most common practiced coping strategies to tackle with anxiety during the quarantine period (Baloran, 2020; Talidong, \& Toquero, 2020). Similar coping styles have been reported in earlier studies where following strict personal preventive behaviors and avoidance of going 
to public places to minimize the risk of infectious have been highly practiced (Faye et al., 2015; Khalid et al., 2016).

Within the wider literature, coping strategies have been found to be associated with a wide range of factors. Adaptive coping (i.e., problems-focused and positive reappraisal coping strategies) has been found to be negatively related with depressive symptoms (Erickson, \& Feldstein, 2007), anxiety (Brouzos, Vassilopoulos, Vlachioti \& Baourda, 2020), and stress (Viana Machado et al., 2020). Maladaptive coping strategies (i.e., emotional and avoidance coping strategies) were negatively associated with psychological well-being and resilience (Mayordomo et al., 2016), humor and adjustment (Erickson, \& Feldstein, 2007), optimism (Scheier, Weintraub, \& Carver, 1986), and self-esteem (Brouzos et al., 2020). Accordingly, identifying coping strategies used in response to infectious disease outbreaks is critical to support the general public in tackling with the pandemic.

\section{Present study}

Given the lack of current empirical evidence on the psychological factors affecting general health in the context of COVID-19, this study aims to contribute to the extant literature in general health during COVID-19 pandemic and incorporates the study of COVID-19 anxiety and coping using a large Turkish sample. Therefore, we investigated whether these potential variables provide explanatory power in the prediction of general health and whether COVID-19 coping explains the underlying mechanism in the relationship between COVID-19 anxiety and general health. This study aimed to examine the associations between COVID-19 anxiety, COVID-19 coping, and general health. Specifically, we hypothesized that (i) COVID-19 anxiety would have a significant direct effect on COVID-19 coping and general health; (ii) COVID-19 coping would significant direct effect on general health; and (iii) COVID-19 coping would function as a significant mediator in the 
relationship between COVID-19 anxiety and general health. The hypothesized structural model is presented in Figure 1.

\section{Method}

\section{Participants}

In all, 4,624 people drawn from the general population aged 18 to 88 years took part in the study. The mean age of participants was $30.29(\mathrm{SD}=10.93)$ years and most were female (70\%), single (58\%), university students/graduate (63\%) and belonging to the medium socio-economic status group (77\%). Participants predominantly reported no history of chronic diseases (86\%). Participation of this study was voluntary, and participants were not given any incentive for their participation.

\section{Measures}

Anxiety. COVID-19 anxiety was measured with five items developed for the purpose of this study. Example item is "I feel anxious about my future due to COVID-19." Items are scored on a five-point scale ranging from 1 (never) to 5 (always), giving a potential range of 5-25. Higher scores indicate greater anxiety associated with COVID-19. Cronbach's alpha for the present sample was $\alpha=.84$.

Coping. For this study, participants were asked to rate four items ("I believe that I have an ability to cope with the COVID-19") on a five-point Likert scale ranging from 1 (strongly disagree) to 5 (strongly agree), giving a potential range of 4-20. Higher scores show higher levels of COVID-19 related coping strategies. Cronbach's alpha for the present sample was $\alpha=.71$.

General health. In this study, general health was assessed with three questions “During COVID-19, how often have you had trouble with sleep?" answered on a five-point 
Likert scale ranging from 1 (never) to 5 (always), giving a potential range of 3-15. Lower scores refer to better health status. Cronbach's alpha for the present sample was $\alpha=.58$.

\section{Procedure}

The data for this study came from a larger project on psychological factors affecting psychosocial and physical health of general public in Turkey during COVID-19 pandemic. All participants completed online versions of the scales as part of questionnaire batteries being conducted for other studies. A consent was obtained from all participants who have been fully instructed about their rights to withdraw from the study at any time points. The questionnaires were presented to the participants in the same order.

\section{Data analysis}

Initially, the means, standard deviations, tests of normality (i.e., skewness and kurtosis) and internal consistency reliability coefficients of the items and scales were estimated. Tests of normality assumptions demonstrated that the data were approximately normally distributed. Tests of skewness and kurtosis for individual items and variables fall within the adequate range of $\leq|3|$ (D’Agostino, Belanger, \& D'Agostino, 1990; Kline, 2015). Exploratory factor analysis (EFA) was conducted to determine the underlying factor structure of all scales created in this study. Correlation analysis was computed to explore the relationships between the measured variables. The regression analysis met the assumptions of normality, independence of errors, and homoscedasticity. Following the recommendations of Baron and Kenny (1986), three regression models were executed to test the mediating effect of the COVID-19 coping on the relationship between the COVID-19 anxiety and general health. Firstly, the regression analysis was conducted to test the predictive effect of COVID19 anxiety on general health. Secondly, the COVID-19 coping was entered into the model to determine its effect on general health. Thirdly, both COVID-19 anxiety and coping were 
simultaneously included in the model to predict general health. If the predictive effect of COVID-19 anxiety on general health was no longer significant after controlling for the effect of COVID-19 coping, this would suggest full mediation. However, if the predictive effect of COVID-19 anxiety on general health remained significant after controlling for the impact of the COVID-19 coping, partial mediation would be drawn. Finally, the Sobel test (Sobel, 1982) was conducted to determine if the indirect effects of COVID-19 anxiety through mediating role of COVID-19 coping was statistically significant. All analyses were carried out using SPSS v24 for Windows.

\section{Results}

\section{Descriptive analysis}

Table 1 presents the means, standard deviations, skewness scores, kurtosis scores, corrected item-total correlations, alpha if the item was deleted, and factor loadings for the three scales. The mean scores for the individual items ranged from 2.69-3.81. In all scales, the corrected item-total correlations for individual items were above 0.35 , suggesting their appropriateness for scale construction (Tabachnick, \& Fidell, 2007). Except one item (.37), all items had factor loadings above .51, representing "fair" to "excellent" (Comrey \& Lee, 1992).

\section{Exploratory factor analysis}

EFA using the principal axis factoring extraction method was performed separately for each of the scales. The factor analysis results yielded Kaiser-Meyer-Olkin (KMO) values of 0.86 for the anxiety $\left(X^{2}=10241,59, p<0.001\right), 0.69\left(X^{2}=3849,68, p<0.001\right)$ for the coping, and $0.63\left(X^{2}=1279,84, p<0.001\right)$ for general health scales. The EFA also supported that for all scales, a one-factor solution was the most appropriate, with a first initial factor producing an Eigenvalue of 3.25 explaining $65 \%$ of the variance for anxiety, 2.15 explaining 
$54 \%$ of the variance for coping, and 1.64 accounting for $55 \%$ of the variance for general health. All items loaded positively on the respective factors.

\section{Correlation analysis}

Correlation analysis was conducted to explore the relationships between the variables (see Table 2). Results showed that COVID-19 anxiety was negatively significantly correlated with COVID-19 coping ( $r=-.27, p<.001)$, and positively significantly correlated with general health $(r=.57, p<.001)$. COVID-19 coping was significantly negatively correlated with general health $(r=-.18, p<.001)$. The correlation coefficients ranged from small to large based on a conventional frame where $.1 \leq r<.3$ presents a small effect, $.3 \leq r<.5$ presents a medium effect, and $r \geq .5$ present a large effect (Cohen, 1998).

\section{Model testing}

Using regression analysis, a path model was constructed whereby the COVID-19 anxiety was exogenous variable, the COVID-19 coping was the mediating variable, and general health was the dependent variable (see Table 3). In Step 1, the direct effect from the COVID-19 anxiety to general health was negative and significant $(\beta=-.57, p<0.01)$. In Step 2, the direct effect of the COVID-19 anxiety on COVID-19 coping was negative and significant $(\beta=-.27, p<0.01)$. In Step 3, both COVID-19 anxiety and coping were entered into the regression model and the results indicated that both of these variables significantly contributed to the prediction of general health by explaining $32 \%$ of total variance. These findings show that COVID-19 coping partially mediated the relationship between COVID-19 anxiety and general health.

A Sobel test was utilized to statistically test the mediating effect of COVID-19 coping on the association between COVID-19 anxiety and general health. The result was statistically significant $(\mathrm{z}=-10.70, p<.01)$. This further supported our hypothesis in respect to mediating 
effect of COVID-19 coping on the COVID-19 anxiety and general health. The structural model is depicted in Figure 1.

\section{Discussion}

While burgeoning research has considerably aimed to determine the prevalence of mental health problems during COVID-19 pandemic (Liu et al., 2020), relatively little research has focused upon the potential factors that explain the underlying mechanism between pandemic related stressors and general health. Accordingly, this study aimed to examine the role of COVID-19 coping in the relationship between COVID-19 anxiety and general health in a representative sample of Turkish population. In general, the findings supported the three main hypotheses which are discussed in more details below.

Firstly, COVID-19 anxiety had significant direct effect on COVID-19 coping and general health, which is parallel with findings from earlier research (Baloran, 2020; Chew et al., 2020; Talidong, \& Toquero, 2020). This suggests that experience of COVID-19 anxiety negatively affects the ability to cope with stress in the context of COVID-19 and general health.

Secondly, COVID-19 coping had significant direct effects on general health. Past research has also showed that coping is an important factor in contributing psychosocial and physical health of individuals (Yıldırım, 2019). Individuals high in adaptive coping strategies experience less depression (Erickson, \& Feldstein, 2007), anxiety (Brouzos et al., 2020), and stress (Viana Machado et al., 2020) and greater psychological well-being (Mayordomo et al., 2016).

Finally, as expected COVID-19 coping acted as a construct to mediate the relationship between COVID-19 anxiety and general health. This result incorporates with the findings of part research (Cappeliez, \& Robitaille, 2010; Cooper, Katona, Orrell, \& Livingston, 2008). 
This implies that when people suffer from COVID-19 related anxiety, it is more likely that they will have inability to cope with stress which in turn lead them to have poor general health. This indeed provides a mechanism that could partially explain why COVID-19 anxiety diminishes general health, with that involving inability to cope with stress. Accordingly, experience of anxiety is likely to contribute to vulnerability to stress in the forms of low capability to deal with stressors which leads to worsen general health. However, it is important to note that mediation analysis suggests longitudinal causality, which cannot be inferred with cross-sectional data (MacKinnon, Fairchild, \& Fritz, 2007).

With regard to the psychometric properties of the scales used in the current study, we found that the one-factor structure of the all three scales has been supported and they had acceptable to good internal consistency coefficients. The scales were also related to one another. The aforementioned scales can be used as screening tools to assess COVID-19 anxiety, COVID-19 general coping strategies, and general health.

This study has several theoretical and practical contributions. In terms of theory, the study expands the predictors of general health through demonstrating COVID-19 coping as a significant explanatory factor in the association between COVID-19 anxiety and general health. Past studies have examined the protective roles of coping strategies in protecting mental health following trauma (Park, Chang, \& You, 2015). This study did not only provide direct evidence that the ability of dealing effectively with difficult situations such as COVID19 can significantly contribute to protect general health, but the study also contributed to our understanding on the mechanism of how COVID-19 anxiety influences general health via COVID-19 coping. While coping strategies have been determined as linking with anxiety to influence health (Eisenberg, Shen, Schwarz, \& Mallon, 2012), findings from this research provide implications for future studies to further examine such mechanism beyond Turkish 
population. Employing different samples is useful, because people use different coping strategies and some people seem to cope with adversity better than others.

In terms of practice, the findings suggest that as COVID-19 anxiety significantly predicts general health through the mediating effect of COVID-19 coping, practitioners are recommended to tailor and implement contextually sensitive interventions and programs that incorporate COVID-19 anxiety as a key element. For instance, using psychological strengthbased interventions in the form of minimizing COVID-19 anxiety may be particularly fruitful for people who experience psychological distress, given that it hinders the ability to cope with stress instead of actively engage in solving the problems. Practitioners are also encouraged to focus on psychological treatments that could strengthen the ability of individuals to manage stressful situations. With this capability, these people may accordingly realize the potential of personal strengths which could potentially mitigate their levels of anxiety.

This study used a cross-sectional design, which limits conclusion from cause-andeffect relation to be inferred. Notwithstanding, the present findings present guidance for future investigations on potential causal relations among the study variables. Another limitation of this study is that participants were largely female, single, university students/graduate, and belonging to the medium socio-economic status group. The generalizability of these findings to other samples with different socio-demographic characteristics is limited. Furthermore, future studies can examine the role of other variables including demographic variables that may influence the associations among the measured variables.

In conclusion, the present study is the first to examine the role of COVID-19 coping in the relationship between COVID-19 anxiety and general health. The results supported the 
notion that COVID-19 coping reduces the impact of COVID-19 anxiety on general health. Therefore, the results underscore a need for researchers and practitioners to develop and implement coping-based intervention strategies to protect the general health of individuals against stressors emerged in difficult times. 


\section{References}

Ahorsu, D. K., Lin, C. Y., Imani, V., Saffari, M., Griffiths, M. D., \& Pakpour, A. H. (2020). The fear of COVID-19 scale: development and initial validation. International Journal of Mental Health and Addiction, 1-9. doi: 10.1007/s11469-020-00270-8

Arslan, G., Yıldırım, M., Tanhan, A., Buluş, M., \& Allen, K. A. (2020). Coronavirus Stress, Optimism-Pessimism, Psychological Inflexibility, and Psychological Health: Testing the Psychometrics of the Coronavirus Stress Measure. International Journal of Mental Health and Addiction, 1-17. Doi: 10.1007/s11469-020-00337-6

Bao, Y., Sun, Y., Meng, S., Shi, J., \& Lu, L. (2020). 2019-nCoV epidemic: address mental health care to empower society. The Lancet, 395(10224), e37-e38.

Baron, R. M., \& Kenny, D. A. (1986). The moderator-mediator distinction in social psychological research: conceptual, strategic, and statistical considerations. Journal of Personality and Social Psychology, 51, 1173-182.

Baloran, E. T. (2020). Knowledge, Attitudes, Anxiety, and Coping Strategies of Students during COVID-19 Pandemic. Journal of Loss and Trauma, 1-8. doi.org/10.1080/15325024.2020.1769300

Brouzos, A., Vassilopoulos, S. P., Vlachioti, A., \& Baourda, V. (2020). A coping-oriented group intervention for students waiting to undergo secondary school transition: Effects on coping strategies, self-esteem, and social anxiety symptoms. Psychology in the Schools, 57(1), 31-43. 
Cappeliez, P., \& Robitaille, A. (2010). Coping mediates the relationships between reminiscence and psychological well-being among older adults. Aging \& Mental Health, 14(7), 807-818.

Carver, C. S., Scheier, M. F., \& Weintraub, J. K. (1989). Assessing coping strategies: a theoretically based approach. Journal of Personality and Social Psychology, 56(2), 267-283.

Center for Systems Science and Engineering. (2020). Coronavirus COVID-19 global cases at Johns Hopkins University. Available from https://coronavirus.jhu.edu/map.html

Chew, Q. H., Wei, K. C., Vasoo, S., Chua, H. C., \& Sim, K. (2020). Narrative synthesis of psychological and coping responses towards emerging infectious disease outbreaks in the general population: practical considerations for the COVID-19 pandemic. Singapore Medical Journal. Advance online publication. ttps://doi.org/10.11622/smedj.2020046

Cohen J. (1988). Statistical power analysis for the behavioral sciences (2nd ed.). Hillsdale, NJ: Erlbaum.

Comrey A. L., \& Lee H. B. (1992). A first course in factor analysis. Hillsdale, NJ: Erlbaum.

Cooper, C., Katona, C., Orrell, M., \& Livingston, G. (2008). Coping strategies, anxiety and depression in caregivers of people with Alzheimer's disease. International Journal of Geriatric Psychiatry: A Journal of the Psychiatry of Late Life and Allied Sciences, 23(9), 929-936.

D’Agostino, R. B., Belanger, A., \& D’Agostino, R. B. (1990). A Suggestion for Using Powerful and Informative Tests of Normality. The American Statistician, 44(4), 31621. 
Eisenberg, S. A., Shen, B. J., Schwarz, E. R., \& Mallon, S. (2012). Avoidant coping moderates the association between anxiety and patient-rated physical functioning in heart failure patients. Journal of Behavioral Medicine, 35(3), 253-261.

Erickson, S. J., \& Feldstein, S. W. (2007). Adolescent humor and its relationship to coping, defense strategies, psychological distress, and well-being. Child psychiatry and Human Development, 37(3), 255-271.

Faye, O., Boelle, P. Y., Heleze, E., Faye, O., Loucoubar, C., Magassouba, N., Soropogui, B., Keita, S., Gakou, T., Bah el, H. I., Koivogui, L., Sall, A. A., \& Cauchemez, S. (2015). Chains of transmission and control of Ebola virus disease in Conakry, Guinea, in 2014: An observational study. The Lancet, 15(3), 320-326. https://doi.org/10.1016/S1473- 3099(14)71075-8

Folkman, S. (2008). The case for positive emotions in the stress process. Anxiety, Stress and Coping, 21(1), 3-14.

Hooley, J. M., Butcher, J. N., Matthew, K. N., \& Mineka, S. (2016). Abnormal psychology. Boston: Pearson.

Khalid, I., Khalid, T., Qabajah, M., Barnard, A., \& Qushmaq, I. (2016). Healthcare workers emotions, perceived stressors and coping strategies during a MERS-CoV outbreak. Clinical Medicine \& Research, 14(1), 7-14. https://doi.org/10.3121/cmr.2016.1303

Kline, R. B. (2015). Principles and practice of structural equation modeling. New York, NY: Guilford.

Lazarus, R. S., \& Folkman, S. (1984). Stress, Appraisal, and Coping. New York: Springer. 
Liu, N., Zhang, F., Wei, C., Jia, Y., Shang, Z., Sun, L., ... \& Liu, W. (2020). Prevalence and predictors of PTSS during COVID-19 outbreak in China hardest-hit areas: Gender differences matter. Psychiatry Research, 287, 112921.

MacKinnon, D. P., Fairchild, A. J., \& Fritz, M. S. (2007). Mediation analysis. Annual Review of Psychology, 58, 593-614.

Maunder, R. G., Lancee, W. J., Balderson, K. E., Bennett, J. P., Borgundvaag, B., Evans, S., ... \& Hall, L. M. (2006). Long-term psychological and occupational effects of providing hospital healthcare during SARS outbreak. Emerging infectious diseases, 12(12), 1924-1932.

Mayordomo, T., Viguer, P., Sales, A., Satorres, E., \& Meléndez, J. C. (2016). Resilience and coping as predictors of well-being in adults. The Journal of Psychology, 150(7), 809821.

Pappas, G., Kiriaze, I. J., Giannakis, P., \& Falagas, M. E. (2009). Psychosocial consequences of infectious diseases. Clinical microbiology and infection, 15(8), 743-747.

Park, M., Chang, E. R., \& You, S. (2015). Protective role of coping flexibility in PTSD and depressive symptoms following trauma. Personality and Individual Differences, 82, $102-106$

Rossi, R., Socci, V., Pacitti, F., Di Lorenzo, G., Di Marco, A., Siracusano, A., \& Rossi, A. (2020). Mental health outcomes among front- and second-line health workers associated with the COVID-19 pandemic in Italy. medRxiv.

Scheier, M. F., Weintraub, J. K., \& Carver, C. S. (1986). Coping with stress: Divergent strategies of optimists and pessimists. Journal of Personality and Social Psychology, 57(6), 1024-1040. 
Sim, K., Chan, Y. H., Chong, P. N., Chua, H. C., \& Soon, S. W. (2010). Psychosocial and coping responses within the community health care setting towards a national outbreak of an infectious disease. Journal of Psychosomatic Research, 68(2), 195202.

Sobel, M. E. (1982). Asymptotic confidence intervals for indirect effects in structural equation models. In S. Leinhardt (Ed.), Sociological methodology (pp. 290-312). Washington DC: American Sociological Association.

Tabachnick, B. G., \& Fidell, L. S. (2007). Using multivariate statistics (5th ed.). New York: Allyn \& Bacon.

Talidong, K. J. B., \& Toquero, C. M. D. (2020). Philippine teachers' practices to deal with anxiety amid COVID-19. Journal of Loss and Trauma, 1-7. doi.org/10.1080/15325024.2020.1759225

The Academy of Medical Sciences. (2020). Survey results: Understanding people's concerns about the mental health impacts of the COVID-19 pandemic. Available at https://acmedsci.ac.uk/file-download/99436893

Viana Machado, A., Volchan, E., Figueira, I., Aguiar, C., Xavier, M., Souza, G. G., ... \& Mocaiber, I. (2020). Association between habitual use of coping strategies and posttraumatic stress symptoms in a non-clinical sample of college students: A Bayesian approach. Plos One, 15(2), e0228661.

Wang, C., Pan, R., Wan, X., Tan, Y., Xu, L., Ho, C. S., \& Ho, R. C. (2020). Immediate psychological responses and associated factors during the initial stage of the 2019 coronavirus disease (COVID-19) epidemic among the general population in China. International Journal of Environmental Research and Public Health, 17(5), 1729. 
Wu, K. K., Chan, S. K., \& Ma, T. M. (2005). Posttraumatic stress, anxiety, and depression in survivors of severe acute respiratory syndrome (SARS). Journal of Traumatic Stress: Official Publication of The International Society for Traumatic Stress Studies, 18(1), $39-42$.

Y1ldırım, M. (2019). Irrational Happiness Beliefs: Conceptualization, Measurement and its Relationship with Well-being, Personality, Coping Strategies, and Arousal. (Unpublished Doctoral Dissertation). University of Leicester, Leicester, UK

Y1ldırım, M., \& Arslan, G. (2020). Exploring the Associations Between Resilience, Dispositional Hope, Subjective Well-Being, and Psychological Health Among Adults During Early Stage of COVID-19, Psyarxiv, 1-27. Preprint doi: 10.31234/osf.io/vpu5q

Yıldırım, M., Geçer, E., \& Akgül, Ö. (2020). The impacts of vulnerability, perceived risk, and fear on preventive behaviours against COVID-19. Psychology, Health \& Medicine, 1-9. Doi: 10.1080/13548506.2020.1776891 
Table 1.

Descriptive statistics of the COVID-19 Anxiety Scale, COVID-19 Coping Scale, and General Health Scale

Table 1. Descriptive statistics of the COVID-19 Anxiety Scale, COVID-19 Coping Scale, and General Health Scale

\begin{tabular}{|c|c|c|c|c|c|c|c|}
\hline Item & M & SD & sk & $\mathrm{ku}$ & $r_{i t}$ & $\alpha_{\mathrm{iid}}$ & FL \\
\hline \multicolumn{8}{|l|}{ COVID-19 Anxiety Scale } \\
\hline 1. I feel anxious about my future due to COVID- 19 . & 3.81 & 0.84 & -0.17 & -0.27 & 0.68 & 0.84 & 0.75 \\
\hline 2. I feel anxious about my health because of COVID-19. & 3.78 & 0.79 & -0.05 & -0.29 & 0.74 & 0.82 & 0.82 \\
\hline 4. I am preoccupied with anxious thoughts of getting infected to coronavirus. & 3.61 & 0.79 & 0.04 & 0.10 & 0.68 & 0.84 & 0.74 \\
\hline 5. I am concerned that coronavirus will affect all aspects of my life. & 3.70 & 0.83 & -0.05 & -0.22 & 0.63 & 0.85 & 0.67 \\
\hline \multicolumn{8}{|l|}{ COVID-19 Coping Scale } \\
\hline 2. I am able to enjoy my life despite the COVID-19. & 2.71 & 0.93 & 0.02 & 0.17 & 0.49 & 0.64 & 0.58 \\
\hline 3. I can get through the COVID- 19 . & 2.69 & 0.81 & -0.15 & 0.84 & 0.61 & 0.57 & 0.81 \\
\hline 4. I can still focus on the meaning of my life even during the COVID-19. & 2.97 & 0.90 & -0.02 & 0.36 & 0.52 & 0.62 & 0.72 \\
\hline \multicolumn{8}{|l|}{ General Health Scale } \\
\hline 1. During COVID-19, how often have you had trouble with sleep? & 2.77 & 0.69 & -0.70 & 2.41 & 0.39 & 0.46 & 0.58 \\
\hline 2. How often have you had difficulty concentrating on your daily routine since COVID-19 had occurred? & 2.77 & 0.83 & -0.17 & 0.77 & 0.37 & 0.52 & 0.51 \\
\hline
\end{tabular}

Note. $\mathrm{M}=$ mean; sk=Skewness; ku=Kurtosis; $\mathrm{r}_{\mathrm{it}}=$ Corrected item-total correlation; $\alpha_{\mathrm{iid}}=$ Cronbach's alpha if item deleted; $F L=$ factor loadings 
Table 2.

Descriptive statistics and correlation results

\begin{tabular}{lcccccccc}
\hline Variable & Mean & SD & Skewness & Kurtosis & $\alpha$ & 1. & 2. & 3. \\
\hline 1. COVID-19 Anxiety & 18.62 & 3.24 & 0.03 & 0.15 & .84 & 1 & $-.27^{* *}$ & $-.57^{* *}$ \\
2. COVID-19 Coping & 11.42 & 2.55 & -0.25 & 1.22 & .71 & 1 & $.18^{* *}$ \\
3. General health & 8.32 & 1.61 & -0.79 & 2.38 & .58 & & 1 \\
\hline
\end{tabular}

Note. $* * p<0.01$ 
Table 3.

Coefficients for the mediating effect

\begin{tabular}{lllcccccc}
\hline Step & Outcome & Predictor & B & SE & $\beta$ & $\mathrm{t}$ & $p$ & $r^{2}$ \\
\hline Step 1 (path c) & General health & Anxiety & -0.28 & 0.01 & -0.57 & -46.93 & 0.00 & 0.32 \\
Step 2 (path a) & Coping & Anxiety & -0.21 & 0.01 & -0.27 & -19.30 & 0.00 & 0.08 \\
Step 3 (path c') & General health & Anxiety & -0.28 & 0.01 & -0.56 & -44.52 & 0.00 & \\
& & Coping & 0.02 & 0.01 & 0.03 & 2.37 & 0.02 & 0.32 \\
\hline
\end{tabular}


Figure 1.

Final schematic diagram in the mediational effects of the COVID-19 coping on the relations between COVID-19 anxiety and general health.

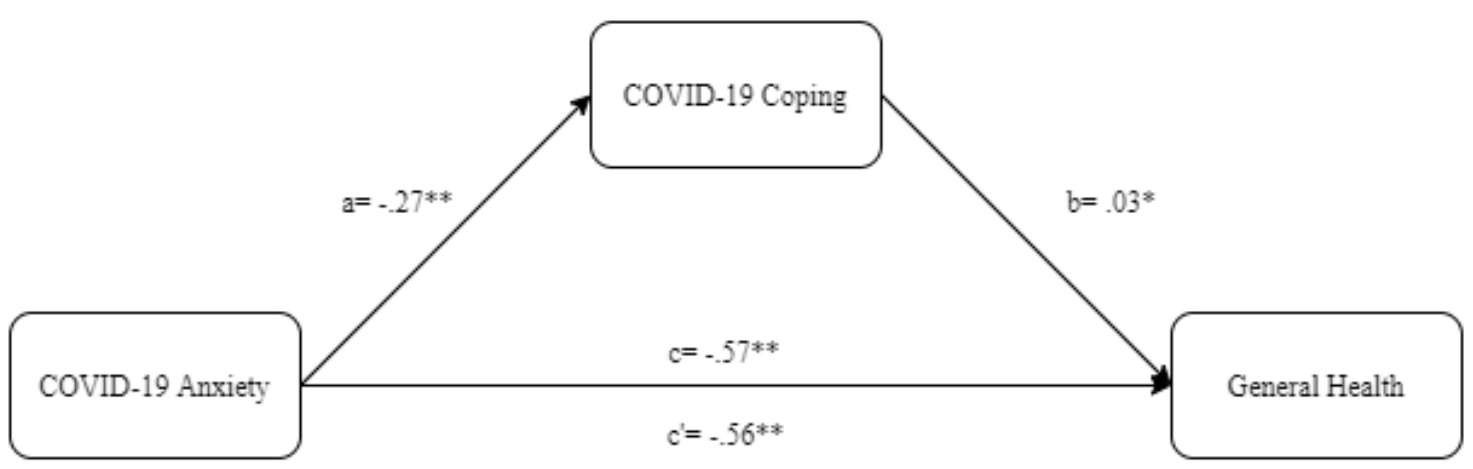

